\title{
Exploring the interparticle electron transfer process in the photocatalytic oxidation of 4-chlorophenol
}

\author{
Hsin-Hung Ou ${ }^{a}$, Shang-Lien $\mathrm{Lo}^{\mathrm{a}}{ }^{\mathrm{*}}$, Chung-Hsin $\mathrm{Wu}^{\mathrm{b}}$ \\ ${ }^{a}$ Research Center for Environmental Pollution Prevention and Control Technology, Graduate Institute of Environmental Engineering, \\ National Taiwan University, 71 Chou-Shan Rd., Taipei 106, Taiwan, ROC \\ ${ }^{\mathrm{b}}$ Department of Environmental Engineering, Da-Yeh University, 112 Shan-Jiau Rd., Da-Tsuen, Chang-Hua 515, Taiwan, ROC
}

Received 6 October 2005; received in revised form 15 March 2006; accepted 15 March 2006

Available online 16 May 2006

\begin{abstract}
This work aimed to investigate the interparticle electron transfer (IPET) process within the coupled-photocatalyst systems on the basis of the degradation of 4-chlorophenol (4-CP). $\mathrm{TiO}_{2}, \mathrm{ZnO}$ and $\mathrm{SnO}_{2}$ are used as the model photocatalysts owing to their increasing energy levels which correspond to the IPET concept. In the single-photocatalyst tests, $\mathrm{ZnO}$ tests are associated with the highest degradation rate constants $\left(0.347 \pm 0.083 \mathrm{~h}^{-1}\right.$ at $\mathrm{pH} 7$ and $0.453 \pm 0.011 \mathrm{~h}^{-1}$ at $\left.\mathrm{pH} 11\right)$ and a better DOC reduction than in other single catalyst tests under given conditions. $\mathrm{ZnO} / \mathrm{SnO}_{2}$ coupled tests have constants of $0.612 \pm 0.068$ and $0.948 \pm 0.069 \mathrm{~h}^{-1}$ at $\mathrm{pH} 7$ and 11 , respectively. Additionally, the $4-\mathrm{CP}$ prefers the breakdown of chloride group in $\mathrm{TiO}_{2}$ system while proceeding hydroxylation reaction in $\mathrm{ZnO}$ systems. Meanwhile, a phenomenonlogical model coupled with the IPET effect was developed to explore the separation of photo-electrons and photo-holes within catalysts. Based on the model parameters, the recombination rate of photo-electrons and photo-holes in $\mathrm{TiO}_{2} / \mathrm{SnO}_{2}$ and $\mathrm{ZnO} / \mathrm{SnO}_{2}$ systems is $20-45 \%$ lower than that obtained by a respective single catalyst. Thus, coupled-photocatalyst tests, $\mathrm{TiO}_{2} / \mathrm{SnO}_{2}$ and $\mathrm{ZnO} / \mathrm{SnO}_{2}$ efficiently suppress the recombination, particularly for $\mathrm{ZnO} / \mathrm{SnO}_{2}$ tests at $\mathrm{pH} 11$.
\end{abstract}

(C) 2006 Elsevier B.V. All rights reserved.

Keywords: Photodegradation; Interparticle electron transfer process; Coupled-photocatalyst

\section{Introduction}

The problem of pollutants, particularly the serious environmental impact of their residues and the relatively low efficiency of the current remediation process, has led to several efforts to elucidate more efficient degradation alternatives. Many works have attempted to degrade numerous pollutants by advanced oxidation processes (AOPs) [1,2], rather than by other treatment processes. Heterogeneous photocatalysis has been emerged as an efficient method for purifying water and air [3,4]. In most cases, the most effective materials for photocatalytic applications are nano-sized semiconductor oxides, which have been proven as excellent catalysts because of their highly reactive surfaces [5]. Such oxides include $\mathrm{TiO}_{2}, \mathrm{ZnO}$ and $\mathrm{CdS}$, among others. $\mathrm{TiO}_{2}$ and $\mathrm{ZnO}$ have been extensively examined as heterogeneous semiconductor photocatalysts, primarily because of

\footnotetext{
* Corresponding author. Fax: +886 223928830 .

E-mail address: sllo@ntu.edu.tw (S.-L. Lo).
}

their high capacity for degrading toxic and recalcitrant chemical species via relatively simple and low-cost procedures $[6,7]$.

However, the use of semiconductors as photocatalysts is mainly limited in the recombination of the generated photoholes and photo-electrons. Photocatalysis involves the oxidation of a chemical by photo-holes from the semiconductor, so every recombination event involves the loss of holes that might otherwise have promoted degradation. Thus, the vectorial transfer of photogenerated electrons and holes between the valence and conduction bands of semiconductors is an important process in photocatalysis. Accordingly, a major focus of current photocatalysis research is to improve the separation characteristics, to improve photo-efficiency. Attempts are made to solve the problem of increasing the photo-efficiency, one of these methods is interparticle electron transfer (IPET) process.

The concept of IPET process is to exploit the distinction of the band gap between semiconductors with different energy potentials to drive the electrons transfer. That means the coupled semiconductors with their corresponding conduction and valence bands can be used to achieve such phenomenon, increasing the 


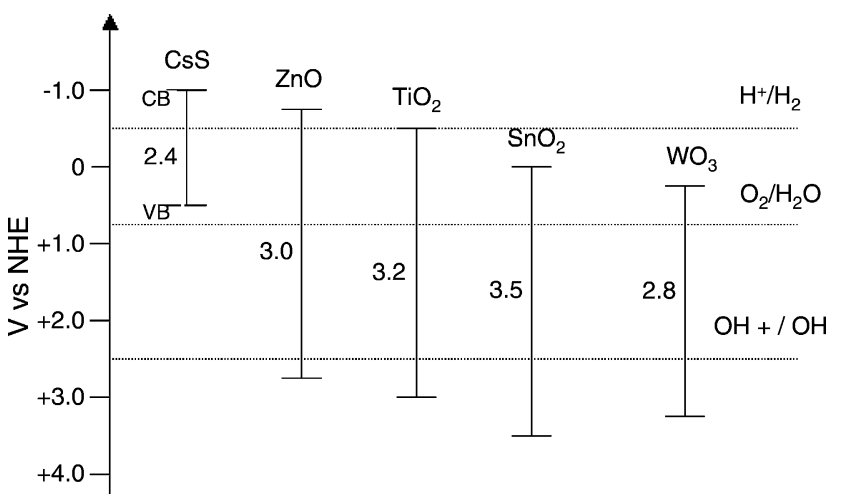

Fig. 1. Diagram depicting the redox potentials of the valence and conduction bands and the band gap energies for various semiconductor particulates $[8,30-33]$.

lifetime of charge carries and the efficiency of the interfacial charge transfer to the adsorbed substrate and ultimately enhancing their photocatalytic performance considerably [8-11]. However, an ambiguous point still needed to be clarified is the promotion in retarding recombination within IPET process. Therefore, it is essential to expound the IPET effect on the basis of some kinetic parameters, and which is the major concern in this study.

This study elucidate the IPET effect under three buffer conditions, $\mathrm{pH} 4,7$ and 11 , using $\mathrm{TiO}_{2}, \mathrm{ZnO}$ and $\mathrm{SnO}_{2}$ as model photocatalysts because of the increase in their energy levels (Fig. 1). Attempts are made to compare the photocatalytic mechanism in the $\mathrm{TiO}_{2}$ and $\mathrm{ZnO}$ systems, by exploiting the reduction of dissolved organic carbon and the ratios of released chloride ions to 4-CP concentration. Additionally, a notional model proposed in the study is used to ascertain whether IPET effect promote the separation of photo-electrons and photoholes.

\section{Materials and methods}

\subsection{Reagent and chemicals}

Three semiconductor powders $\mathrm{TiO}_{2}$ (Degussa P25), $\mathrm{ZnO}$ (Fluka) and $\mathrm{SnO}_{2}(\mathrm{RDH})$ were used as model photocatalysts. 4-CP (99\%) was purchased from Aldrich and was of the best grade available; these agents were used without further treatment. Water was deionized and doubly distilled with Milli-Q.

\subsection{Experimental apparatus and procedures}

All photodegradation experiments were conducted in a batch reactor. The reaction mixtures were illuminated under a UV lamp (8 W Xenon lamp, Philips), which was placed approximately $35 \mathrm{~cm}$ below the bottom of the glass plate at a controlled reaction temperature of $25^{\circ} \mathrm{C}$ during the experimental period. The intensity of UV lamp is $4.32 \mathrm{~mW} \mathrm{~cm}^{-2}$ measured by UV radiometer (MS-100, UVP) at the sampling position.

Prior to the photocatalytic experiments, the suspensions were prepared by mixing definite volumes $(3 \mathrm{~L})$ of solutions of the desired concentration of 4 - $\mathrm{CP}\left(2 \times 10^{-4} \mathrm{M}\right)$ with various sin-
Table 1

Characteristic property of $\mathrm{TiO}_{2}, \mathrm{ZnO}$ and $\mathrm{SnO}_{2}$

\begin{tabular}{lllc}
\hline Catalyst & $\begin{array}{l}\text { Maximum absorption } \\
\text { wavelength }(\mathrm{nm})\end{array}$ & Band gap $(\mathrm{eV})$ & BET $\left(\mathrm{m}^{2} \mathrm{~g}^{-1}\right)$ \\
\hline $\mathrm{TiO}_{2}$ & 391.4 & 3.17 & 54.18 \\
$\mathrm{ZnO}$ & 424.9 & 2.92 & 4.56 \\
$\mathrm{SnO}_{2}$ & 300.4 & 4.13 & 4.78 \\
\hline
\end{tabular}

gle and coupled-photocatalysts, $\mathrm{TiO}_{2}, \mathrm{ZnO}, \mathrm{SnO}_{2}, \mathrm{TiO}_{2} / \mathrm{SnO}_{2}$, $\mathrm{ZnO} / \mathrm{SnO}_{2}$ and $\mathrm{TiO}_{2} / \mathrm{ZnO}\left(1.2 \mathrm{~g} \mathrm{~L}^{-1}\right)$. Meanwhile, the coupled catalysts are of physical mixing in the solution without any pretreatments. The portion of $\mathrm{SnO}_{2}$ is $50 \mathrm{wt}$ \% for respective coupled catalyst. That means both the concentrations of $\mathrm{TiO}_{2}$ and $\mathrm{SnO}_{2}$ in the coupled catalyst $\left(\mathrm{TiO}_{2} / \mathrm{SnO}_{2}\right)$ are $0.6 \mathrm{~g} \mathrm{~L}^{-1}$, the proportion of $\mathrm{SnO}_{2}$ in $\mathrm{ZnO} / \mathrm{SnO}_{2}$ coupled catalyst is the same with the aforementioned description. The slurry was stirred magnetically for $30 \mathrm{~min}$ in the dark to achieve adsorption equilibrium for the substrate on the photocatalytic system. Hydrochloric acid or sodium hydroxide was added to maintain the desired buffered conditions. When the photocatalytic experiment was initiated, the samples were withdrawn at different times. After they were centrifuged at $3000 \mathrm{rpm}$ with a centrifuge (KUBOTA KN-70) and then filtered through a $0.45 \mu \mathrm{m}$ filter membrane (Millipore), the samples proceeded to a series of analysis, the remaining of 4CP (HPLC, Millipore Waters 600E with a Waters 486 detector), the quantification of released chloride ions (IC, Dionex model DX-120) and dissolved organic carbon (TOC, 1020A, OI Analytical). And all the physical characteristics of the photocatalysts performed in the study were presented in Table 1 .

\section{Model development}

Although the production of by-products such as hydroquinone (HQ), benzoquinone (BQ) and 4-chlorocatechol (4-CC) can potentially influence the degradation behavior of 4-CP, their impacts have been inclusive of the model development. The model primarily concerns the type of coupled-photocatalystic systems and the reaction conditions to survey the IPET effect. The simplified notional mechanism of photocatalysis is described as follows:

$$
\begin{aligned}
& \mathrm{TiO}_{2}, \mathrm{ZnO}+h v \rightarrow \mathrm{e}^{-}+h^{+} \\
& \mathrm{e}^{-}+\mathrm{SnO}_{2} \rightarrow \mathrm{e}^{-} \cdot \mathrm{SnO}_{2} \\
& \mathrm{e}^{-}+\mathrm{h}^{+} \rightarrow \text { heat } \\
& \mathrm{e}^{-}+\mathrm{O}_{2, \text { ads }} \rightarrow \mathrm{O}_{2}^{-} \\
& h^{+}+\mathrm{H}_{2} \mathrm{O}_{\text {ads }} \rightarrow \mathrm{OH}^{\bullet}+\mathrm{H}^{+} \\
& h^{+}+\mathrm{Sub}_{\text {ads }} \rightarrow R^{\bullet}+\mathrm{H}_{2} \mathrm{O}
\end{aligned}
$$

Eq. (1) shows the formation of photo-holes and photoelectrons in the surface of $\mathrm{TiO}_{2}$ or $\mathrm{ZnO}$ on irradiation with the valid UV light energy which is significantly affected by the optical and physical properties of irradiated catalysts. Eq. (2) refers to the vectorial transfer of photo-electrons between two catalysts owing to the distinct energy potential within 
the coupled systems. The reaction channel is so-called IPET process which can reduce the recombination opportunities of photo-holes and photo-electrons. Therefore, such a route theoretically provides an effective approach to conduce on the photocatalytic performance. Meanwhile, Eq. (3) represents the recombination rate of electrons and holes occurred on the surface of the irradiated photocatalysts. The reaction channel is always taken as the major limiting factor on photocatalytic efficiency resulting to the decline of the yields of photo-holes. Another pathway for photo-electrons is the trapping reaction by oxygen molecules, which has been confirmed as the major channel for photo-holes in the photocatalysis [12], such as shown in Eq. (4). Eqs. (5) and (6) demonstrate the channels for the photo-holes to react with the molecular water and target compound, respectively. This study envisages that the photoholes are the prevailing oxidant species on the basis of some prior investigations [13-15]. That means there is a hypothesis, $k_{6} K_{\text {sub }} C_{\text {sub }}>>k_{5} K_{\mathrm{H}_{2} \mathrm{O}} C_{\mathrm{H}_{2} \mathrm{O}}$, where the $K_{\mathrm{H}_{2} \mathrm{O}}$ and $C_{\mathrm{H}_{2} \mathrm{O}}$ are adsorption constant and concentration of water molecules in the system. Regarding the validity of the hypothesis, some typical kinetics from literature provided solid evidences to support that. Sun and Bolton [16] even evaluated the formation rates of $\mathrm{OH}^{\bullet}\left((3.40 \pm 0.13) \times 10^{-7} \mathrm{M} \mathrm{s}^{-1}\right)$ in which $\mathrm{HCHO}$ was chosen as the trapping reagent in the $\mathrm{TiO}_{2}$ suspensions. Ishibashi et al. [15] quantified the quantum yields of hydroxyl radicals and photogenerated holes which were estimated to be $7 \times 10^{-5}$ and $5.7 \times 10^{-2}$, respectively. This implies that oxidative reactions on $\mathrm{TiO}_{2}$ photocatalyst occur mainly via photogenerated holes instead of $\mathrm{OH}^{\bullet}$. Accordingly, the intrinsic photocatalytic kinetic of organic substrate in aqueous slurry can be described as Eq. (7). The detail of the similar model development discussed herein is also presented in the previous studies [5,17-19]:

$\frac{\mathrm{d} C_{\text {sub }}}{\mathrm{d} t}=-k_{6} C_{h+} C_{\text {sub,ads }}$

where the $C_{h^{+}}$and $C_{\text {sub,ads }}$ are the concentration of photoholes and the target compounds on the surface of the $\mathrm{TiO}_{2}$, respectively. Prior to deduce the phenomenonlogical model, one need to suggest the photo-holes and photo-electrons obey the pseudo-steady-state assumption meaning for the results, $\frac{\mathrm{d} C_{\mathrm{e}^{-}}}{\mathrm{d} t}=0$ and $\frac{\mathrm{d} C_{\mathrm{h}}+}{\mathrm{d} t}=0$ [17]. That indicates that the photo-holes and photo-electrons may have constant concentration in an aqueous solution with constant dissolved oxygen concentration, just as shown in Eqs. (8) and (9):

$$
\begin{aligned}
& \frac{\mathrm{d} C_{\mathrm{e}^{-}}}{\mathrm{d} t}=g_{\mathrm{ave}}-\left(k_{3}-k_{2}\right) C_{\mathrm{e}^{-}} C_{\mathrm{h}^{+}}-k_{4} C_{\mathrm{e}^{-}} C_{\mathrm{O}_{2}, \mathrm{ads}} \approx 0, \\
& C_{\mathrm{e}^{-}}=\frac{g_{\mathrm{ave}}}{\left(k_{3}-k_{2}\right) C_{\mathrm{h}^{+}}+k_{4} C_{\mathrm{O}_{2}} K_{\mathrm{O}_{2}}}
\end{aligned}
$$

$$
\begin{aligned}
\frac{\mathrm{d} C_{\mathrm{h}^{+}}}{\mathrm{d} t}= & g_{\mathrm{ave}}-\left(k_{3}-k_{2}\right) C_{\mathrm{e}^{-}} C_{\mathrm{h}^{+}}-k_{5} C_{\mathrm{h}^{+}} C_{\mathrm{H}_{2} \mathrm{O}, \text { ads }} \\
& -k_{6} C_{\mathrm{h}^{+}} C_{\text {sub, ads }} \approx 0, \\
C_{\mathrm{h}^{+}}= & \frac{g_{\text {ave }}}{\left(k_{3}-k_{2}\right) C_{\mathrm{e}^{-}}+k_{5} C_{\mathrm{H}_{2} \mathrm{O}} K_{\mathrm{H}_{2} \mathrm{O}}+k_{6} C_{\mathrm{sub}} K_{\mathrm{sub}}}
\end{aligned}
$$

In the above expressions, $C_{\mathrm{e}^{-}}$and $C_{\mathrm{h}^{+}}$represented the concentration of the photo-electrons and photo-holes generated in each test, respectively. $K_{\mathrm{O}_{2}}$, and $K_{\text {sub }}$ stand for the adsorption constants of oxygen and target compound. $k_{i}$ is the reaction rate for the respective aforementioned reactions. $g_{\text {ave }}$ is the average generated rate of electron-hole pairs in the reaction systems which is dependent on the physical character of $\mathrm{TiO}_{2}$ and $\mathrm{ZnO}$ and the properties of UV light, but it is treated as a lumped parameter in this model. Among the parameters, $C_{\mathrm{H}_{2} \mathrm{O}}$ and $C_{\mathrm{O}_{2}}$ are referred to the concentrations of molecular water and oxygen in the slurry systems. Substituting Eq. (8) into Eq. (9) will derive the quadratic expression for the concentration of the photo-holes adhered to the $\mathrm{TiO}_{2}$ surface, just as shown in Eq. (10):

$$
\begin{gathered}
C_{\mathrm{h}^{+}}^{2}+\frac{\left(k_{4} C_{\mathrm{O}_{2}} K_{\mathrm{O}_{2}} k_{5} C_{\mathrm{H}_{2} \mathrm{O}} K_{\mathrm{H}_{2} \mathrm{O}}+k_{4} C_{\mathrm{O}_{2}} K_{\mathrm{O}_{2}} k_{6} C_{\mathrm{sub}} K_{\mathrm{sub}}\right)}{\left(k_{3}-k_{2}\right)\left(k_{5} C_{\mathrm{H}_{2} \mathrm{O}} K_{\mathrm{H}_{2} \mathrm{O}}+k_{6} C_{\mathrm{sub}} K_{\mathrm{sub}}\right)} C_{\mathrm{h}^{+}} \\
-\frac{g_{\mathrm{ave}} k_{4} C_{\mathrm{O}_{2}} K_{\mathrm{O}_{2}}}{\left(k_{3}-k_{2}\right)\left(k_{5} C_{\mathrm{H}_{2} \mathrm{O}} K_{\mathrm{H}_{2} \mathrm{O}}+k_{6} C_{\mathrm{sub}} K_{\mathrm{sub}}\right)}=0
\end{gathered}
$$

Let, $\quad a=k_{4} C_{\mathrm{O}_{2}} K_{\mathrm{O}_{2}}, b=k_{5} C_{\mathrm{H}_{2} \mathrm{O}} K_{\mathrm{H}_{2} \mathrm{O}}, \quad c=k_{6} K_{\mathrm{sub}}, \quad \sigma=$ $\left(k_{3}-k_{2}\right)$, and then make the assumption which $k_{6} K_{\text {sub }} C_{\text {sub }}>>$ $k_{5} K_{\mathrm{H}} C_{\mathrm{H}_{2} \mathrm{O}}$ indicating that the major portion of photo-holes react with target compounds instead of the molecular water. That means the reaction rate between photo-holes and target compounds is higher than the rate of hydroxyl-radicals-producing which reflects that the photo-holes may be the predominant oxidant species corresponding to the assumption in the study. Such concepts have been held true in some early studies [13-15]. The solution to Eq. (10) is shown as Eq. (11) and then arrange as the following form by substituting it into Eq. (7), and the outcome is showed in Eq. (12):

$$
\begin{aligned}
& C_{\mathrm{h}^{+}}= \frac{-\left(a b+a c C_{\mathrm{sub}}\right)}{2\left(a b+a c C_{\mathrm{sub}}\right)^{2}+4 \sigma g_{\mathrm{ave}}\left(a b+a c C_{\mathrm{sub}}\right)} \\
&= \frac{-a c C_{\mathrm{sub}}+\sqrt{\left(a c C_{\mathrm{sub}}\right)^{2}+4 \sigma g_{\mathrm{ave}}\left(a c C_{\mathrm{sub}}\right)}}{2 \sigma\left(c C_{\mathrm{sub}}\right)} \\
&= \frac{-a}{2 \sigma}+\frac{\sqrt{\left(a c C_{\mathrm{sub}}\right)^{2}+4 \sigma g_{\mathrm{ave}}\left(a c C_{\mathrm{sub}}\right)}}{2 \sigma\left(c C_{\mathrm{sub}}\right)} \\
& \frac{\mathrm{d} C_{\mathrm{sub}}}{\mathrm{d} t}=\left\{\frac{a c}{2 \sigma} C_{\mathrm{sub}}-\frac{a c}{2 \sigma} \sqrt{C_{\mathrm{sub}}^{2}+\frac{4 \sigma g_{\mathrm{ave}}}{a c} C_{\mathrm{sub}}}\right\}
\end{aligned}
$$

Then to expand the term, $\sqrt{C_{\text {sub }}^{2}+\left(4 \sigma g_{\text {ave }} / a c\right) C_{\text {sub }}}$, as the Taylor series expansion with third order which leads to the results shown below and then to arrange as the Eq. (13) followed by some simplifications:

$$
\begin{aligned}
\frac{\mathrm{d} \theta}{\mathrm{d} t}= & 180\left\{\frac{\alpha}{2 \beta} \theta-\frac{\alpha^{1 / 2}}{\beta^{1 / 2}} \gamma^{-(1 / 2)} \theta^{1 / 2}-\frac{1}{8} \frac{\alpha^{3 / 2}}{\beta^{3 / 2}} \gamma^{1 / 2} \theta^{3 / 2}\right. \\
& \left.+\frac{1}{128} \frac{\alpha^{5 / 2}}{\beta^{5 / 2}} \gamma^{3 / 2} \theta^{5 / 2}-\frac{1}{1024} \frac{\alpha^{7 / 2}}{\beta^{7 / 2}} \gamma^{5 / 2} \theta^{7 / 2}\right\}
\end{aligned}
$$

where $\theta=\frac{C_{\text {sub }}}{C_{0}}, \quad \tau=\frac{t}{t_{\mathrm{all}}}, \alpha=c, \beta=\frac{\sigma}{a}$, and $\gamma=\frac{C_{0}}{g_{\mathrm{ave}}}$ 
Eq. (13) is used to fit the experimental data, and the values of kinetic parameters, $\alpha, \beta$ and $\gamma$ are obtained to compare the distinction of chemical behavior among systems as the function of $\mathrm{pH}$ and the IPET process. Accordingly, one can elucidate the IPET effect on observation of the variance of $\beta$ value while $\alpha$ is the observed degradation rate constant. $\gamma$ is greatly dependent on the physical and optical properties of the irradiated photocatalysts, $\mathrm{TiO}_{2}$ and $\mathrm{ZnO}$.

\section{Results and discussions}

\subsection{Background experiments}

The direct photolysis, evaporation of 4-CP free of catalysts in the solution and adsorption of 4-CP on catalysts, need to be performed to exclude the degradation of 4-CP caused by the ambient environment during photocatalysis course. Before that, the optimum amount of catalyst should be ascertained by conducting a series of tests. Fig. 2 plots the obtained initial photodegradation rate constants for different loading amount of $\mathrm{TiO}_{2}$ and the results of background experiments under neutral conditions. Accordingly, the optimal dosage of $\mathrm{TiO}_{2}$ was $1.2 \mathrm{~g} \mathrm{~L}^{-1}$ in all experiments. The results of the background experiments revealed that the disappearance of 4-CP caused by ambient conditions can be negligible, suggesting that the ambient condition had almost no effect on the degradation of 4-CP. Thus, the disappearance of 4-CP in the photocatalytic experiments could be definitely attributed to the photodegradation.

\subsection{Effect of $p H$ and IPET process}

The observed rate constants of 4-CP, the values of $\alpha$ in Table 2, at pH 7 for the $\mathrm{TiO}_{2} / \mathrm{SnO}_{2}$ and $\mathrm{ZnO} / \mathrm{SnO}_{2}$ tests are $0.186 \pm 0.070$ and $0.612 \pm 0.068 \mathrm{~h}^{-1}$, respectively. The corresponding values in the $\mathrm{TiO}_{2}$ and $\mathrm{ZnO}$ tests are $0.127 \pm 0.015$ and $0.347 \pm 0.083 \mathrm{~h}^{-1}$, indicating that the photocatalytic per-

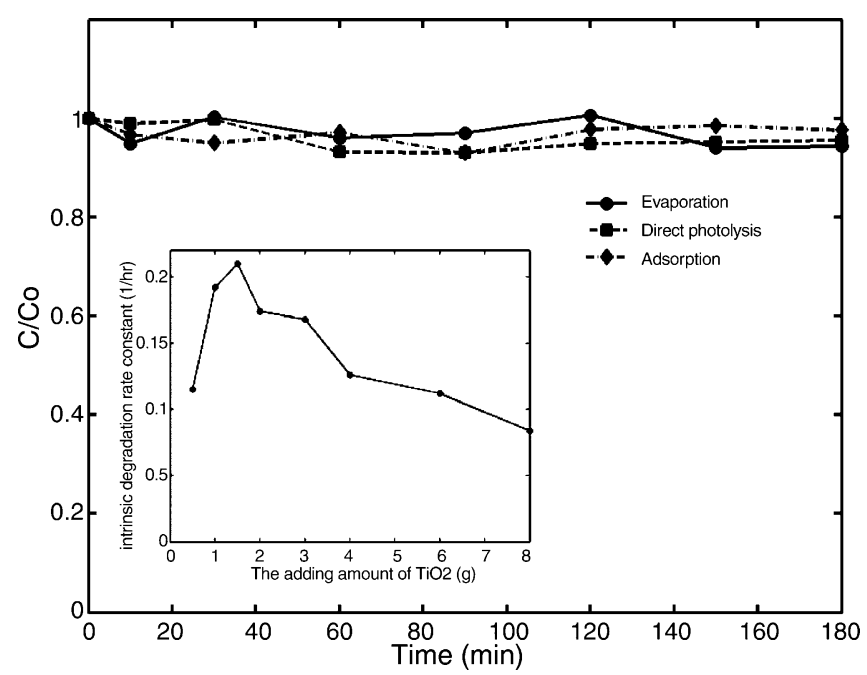

Fig. 2. The effects of background experiments inclusive of the tests of the optimum adding amounts at its neutral condition $\left([4-\mathrm{CP}]_{0}=200 \mu \mathrm{M}\right.$, $\left.\left[\mathrm{TiO}_{2}\right]=1.2 \mathrm{~g} \mathrm{~L}^{-1}\right)$.
Table 2

Comparisons of observed degradation rate constants and model parameters for 4-CP degradation for each system

\begin{tabular}{lclll}
\hline Tests & $\begin{array}{l}\text { Conditions } \\
(\mathrm{pH})\end{array}$ & \multicolumn{4}{l}{ Model parameters } \\
\cline { 3 - 5 } & & $\alpha\left(\mathrm{h}^{-1}\right)$ & $\beta\left(10^{-3}\right)$ & $\gamma\left(10^{-3}\right)$ \\
\hline $\mathrm{TiO}_{2}$ & 4 & $0.109 \pm 0.015$ & $2.257 \pm 0.741$ & $4.005 \pm 0.304$ \\
& 7 & $0.127 \pm 0.015$ & $0.626 \pm 0.049$ & $18.98 \pm 2.379$ \\
& 11 & $0.100 \pm 0.012$ & $0.419 \pm 0.037$ & $49.04 \pm 6.742$ \\
$\mathrm{TiO}_{2} / \mathrm{SnO}_{2}$ & 4 & $0.174 \pm 0.012$ & $1.580 \pm 0.152$ & $0.152 \pm 0.009$ \\
& 7 & $0.186 \pm 0.070$ & $0.427 \pm 0.051$ & $66.92 \pm 2.882$ \\
& 11 & $0.150 \pm 0.011$ & $0.332 \pm 0.028$ & $60.53 \pm 4.191$ \\
$\mathrm{ZnO}$ & 7 & $0.347 \pm 0.083$ & $9.321 \pm 0.881$ & $260.4 \pm 15.31$ \\
& 11 & $0.453 \pm 0.011$ & $4.660 \pm 0.346$ & $392.7 \pm 17.96$ \\
$\mathrm{ZnO} / \mathrm{SnO}_{2}$ & 7 & $0.612 \pm 0.068$ & $5.450 \pm 0.032$ & $255.1 \pm 15.32$ \\
& 11 & $0.948 \pm 0.069$ & $3.494 \pm 0.119$ & $602.1 \pm 31.17$ \\
\hline
\end{tabular}

formance of the coupled-photocatalyst tests is better than that of the single-photocatalyst tests, indirectly confirming the IPET effect. Also expected, the same results are observed at $\mathrm{pH} 11$, at which the observed rate constants in the $\mathrm{TiO}_{2}, \mathrm{TiO}_{2} / \mathrm{SnO}_{2}$, $\mathrm{ZnO}$ and $\mathrm{ZnO}_{2} / \mathrm{SnO}_{2}$ tests are $0.100 \pm 0.012,0.150 \pm 0.011$, $0.453 \pm 0.011$ and $0.948 \pm 0.069 \mathrm{~h}^{-1}$, respectively.

Such finding is consistent with the concept of the IPET process. The $\mathrm{TiO}_{2} / \mathrm{SnO}_{2}$ and $\mathrm{ZnO} / \mathrm{SnO}_{2}$ tests significantly differ in redox energy levels and so exhibit better results concerning the IPET effect, especially for $\mathrm{ZnO} / \mathrm{SnO}_{2}$ tests in which the energy gap is large (Fig. 1). Notably, $\mathrm{TiO}_{2} / \mathrm{ZnO}$ tests dose not deliver the anticipated results, even though the IPET effect is exhibited. This event is associated with the small energy gap between $\mathrm{TiO}_{2}$ and $\mathrm{ZnO}$. Accordingly, $\mathrm{TiO}_{2} / \mathrm{ZnO}$ tests may offer more opportunities for photo-electrons and photo-holes to recombine, reducing the IPET efficiency below that in the $\mathrm{TiO}_{2} / \mathrm{SnO}_{2}$ and $\mathrm{ZnO} / \mathrm{SnO}_{2}$ tests. Although the IPET effect is evident, it is stronger at $\mathrm{pH}$ 11 for $\mathrm{ZnO}$ systems but weaker at $\mathrm{pH} 11$ for $\mathrm{TiO}_{2}$ systems. The results obtained at $\mathrm{pH} 11$ agree with those obtained in most studies, in which highly alkaline media have been shown to promote photocatalysis $[8,20]$.

Notably, the catalytic capacity of $\mathrm{TiO}_{2}$ follows the order $\mathrm{pH}$ $7>\mathrm{pH} 4>\mathrm{pH} 11$. For $\mathrm{TiO}_{2}$ systems at $\mathrm{pH} 4$ and 7 , the predominant species in the solution are the undissociated 4-CP molecules, rather than the corresponding anions $\left(\mathrm{p} K_{\mathrm{a}}=9.2\right)$. Meanwhile, positively charged $\mathrm{TiO}_{2}$, had an acidic surface at $\mathrm{pH} 4$ and 7, which is an effective environment for adsorption, under which the affinity between $\mathrm{TiO}_{2}$ and 4-CP is higher than at $\mathrm{pH} 11$, resulting in the higher degradation rate at $\mathrm{pH} 7$. Also, $\mathrm{Ku}$ and Jung [21] found the surface charge of Degussa P25 in aqueous solution; the $\mathrm{pH}$ at the zero point of charge $\left(\mathrm{pH}_{\mathrm{ZPC}}\right)$ was determined to be 7.52. The aforementioned statements support the result related to the characteristics of $\mathrm{TiO}_{2}$.

Regarding the $\mathrm{ZnO}$ systems, the values of observed rate constant, $\alpha$, are $0.612 \pm 0.068$ and $0.948 \pm 0.069 \mathrm{~h}^{-1}$ at $\mathrm{pH} 7$ and 11 , respectively. The point worthy to mention is that the results indicate alkaline conditions promote photocatalytic performance, which finding is in contrast with the prior expressions. Eqs. (5) and (6) demonstrate a competition between the molecular water and the target compound for photo-holes. Subsequently, 
Eq. (5) theoretically dominates under alkaline conditions. The degradation efficiency in acid condition theoretically exceeds that in alkaline condition. The hypothesis of $k_{6} K_{\text {sub }} C_{\text {sub }}>>$ $k_{5} K_{\mathrm{H}} C_{\mathrm{H}_{2} \mathrm{O}}$ gives a good account of why the opposite events occur. Namely, Eq. (5) is just a minor channel for photo-holes to proceed even in the alkaline conditions.

The results also reveal that $\mathrm{ZnO}$ is a poor photocatalyst in the oxidative degradation of 4-CP at $\mathrm{pH} 4$, because it corrodes in aqueous acidic media, which finding was consistent with that of an earlier literature [22]. Also as expected, $\mathrm{SnO}_{2}$ is not to be inactivated because of the low overlap between the UV emission spectrum (the maximum emission wavelength: $365 \mathrm{~nm}$ ) and adsorption spectrum of $\mathrm{SnO}_{2}$ (the maximum adsorption wavelength: $300.4 \mathrm{~nm}$ ). Interestingly, the activity pattern of 4-CP is $\mathrm{ZnO}>\mathrm{HO}_{2}>\mathrm{SnO}_{2}$ at $\mathrm{pH} 7$ and $11 . \mathrm{ZnO}$ photocatalyst has an activity that is 1.9 times higher than that of $\mathrm{TiO}_{2}$, which is the reference photocatalyst at $\mathrm{pH} 7$ and almost three times that of $\mathrm{TiO}_{2}$ at $\mathrm{pH} 11 . \mathrm{ZnO}$ is concluded to be a good photocatalyst of the degradation of 4-CP in alkali aqueous media, suggesting that $\mathrm{ZnO}$ is highly active in 4-CP degradation. The same results have been published in the previous researches. [23,24].

When $\mathrm{TiO}_{2}$ or $\mathrm{ZnO}$ are coupled with $\mathrm{SnO}_{2}$, the oxidation of 4$\mathrm{CP}$ is faster than that in $\mathrm{TiO}_{2}$ and $\mathrm{ZnO}$ single-tests, respectively, reflecting the charge transfer between $\mathrm{TiO}_{2}$ or $\mathrm{ZnO}$ and $\mathrm{SnO}_{2}$ [8]. The phenomenon indicates that $\mathrm{TiO}_{2}$ and $\mathrm{ZnO}$ are active in 4-CP oxidation but $\mathrm{SnO}_{2}$ is not. However, $\mathrm{SnO}_{2}$ may be a charge carrier in the IPET process while coupled with $\mathrm{TiO}_{2}$ and $\mathrm{ZnO}$. Several studies have involved analogous physical experiments and have found the same trend in the degradation of compounds using coupled-photocatalyst [9-11,25].

\subsection{Mineralization and release of chloride ions}

Many studies have reported the appearance of by-products in the solution, while 4-CP was treated photocatalytically; these are dominantly hydroquinone (HQ), benzoquinone (BQ) and 4chlorocatechol (4-CCT) [8,26-29]. In this work, the reaction pathway in the $\mathrm{TiO}_{2}$ and $\mathrm{ZnO}$ systems is surveyed from the released chloride ions and the extent of mineralization. However, attempts to study the scenario of DOC (dissolved organic carbon) degradation have been inconclusive, because the results have been widely scattered. The ratio of released chloride ions to degraded $4-\mathrm{CP}\left(\left[\mathrm{Cl}^{-}\right]_{\text {formed }} /[4-\mathrm{CP}]_{\text {degraded }}\right)$ and the degree of mineralization are plotted as Figs. 3-5.

Obviously, the initial conversions of 4-CP to chloride ions in $\mathrm{ZnO}$ systems are lower than unity regardless of the conditions (Figs. 4 and 5). This fact clearly indicates that the initial reaction causes the significant collapse of phenyl groups instead of chloride groups. Therefore, the predominant intermediates in $\mathrm{ZnO}$ systems are probably chlorinated organic matters. Despite the better performance of $\mathrm{ZnO}$ systems in 4-CP degradation, most of the corresponding by-products are still highly toxic. In contrast, the ratio of the released chloride ions to degraded 4$\mathrm{CP}$ is lower in $\mathrm{ZnO}$ systems than in $\mathrm{TiO}_{2}$ systems, in which it is approximately unity after $50 \mathrm{~min}$. Subsequently, the initial reactions in the $\mathrm{TiO}_{2}$ systems probably involve the breaking off of the chlorinated function group. That means the by-products

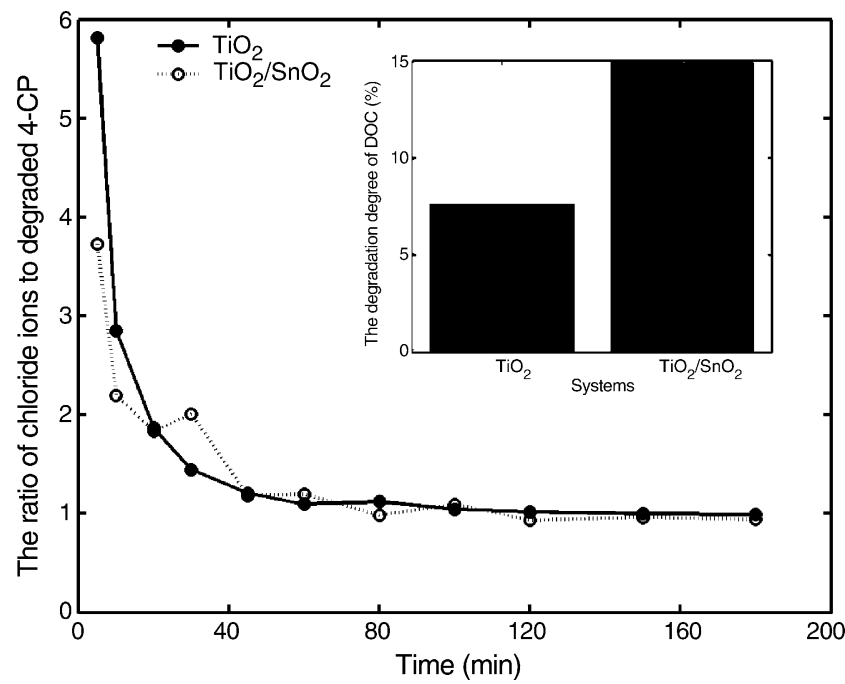

Fig. 3. The scenario of ratios of released chloride ions to the degraded 4-CP inclusive of the reduction of DOC at $\mathrm{pH} 4\left(\left[\mathrm{Cl}^{-}\right]_{\text {formed }} /[4-\right.$ $\left.\mathrm{CP}]_{\text {degraded }}\right) \quad\left([4-\mathrm{CP}]_{0}=200 \mu \mathrm{M}, \quad[\mathrm{DOC}]_{0}=14.4 \mathrm{ppm}, \quad\left[\mathrm{TiO}_{2}\right]=1.2 \mathrm{~g} \mathrm{~L}^{-1}\right.$, $\left.\left[\mathrm{TiO}_{2} / \mathrm{SnO}_{2}\right]=0.6 / 0.6 \mathrm{~g} \mathrm{~L}^{-1}\right)$.

produced in $\mathrm{TiO}_{2}$ system prefer $\mathrm{HQ}$ and $\mathrm{BQ}$ instead of 4-CC. Stafford et al. [26] have indicated the increased $\mathrm{TiO}_{2}$ loading $\left(0.025 \sim 1.000 \mathrm{~g} \mathrm{~L}^{-1}\right)$ significantly decreases the 4 -CC concentration. This observation corresponded to the aforementioned inference, where the $\mathrm{TiO}_{2}$ loading in our study was $1.2 \mathrm{gL}^{-1}$. Alike phenomenon has also been reported by Alekabi and Serpone [27] and Alsayyed et al. [28]. Regarding the by-products produced in $\mathrm{ZnO}$ system, the majority of the by-products may be 4-CC [29], which is the predominant reason leading to the ratios, the released chloride ions to the degraded 4-CP, are lower than unity.

Notably, the disappearance of DOC shows that decarboxylation is an initial step in $\mathrm{ZnO}$ photo-degradation, since the initial rate $(-\mathrm{d}[\mathrm{DOC}] / \mathrm{d} t)$ was not zero which is not showed in the

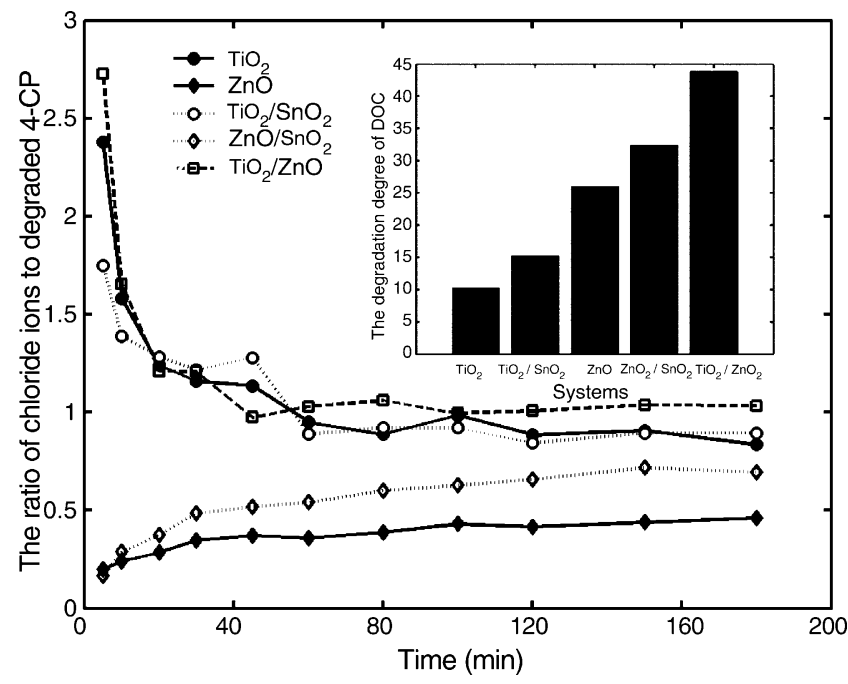

Fig. 4. The scenario of ratios of released chloride ions to the degraded 4-CP inclusive of the reduction of DOC at $\mathrm{pH} 7\left(\left[\mathrm{Cl}^{-}\right]_{\text {formed }} /[4-\mathrm{CP}]_{\text {degraded }}\right)\left([4-\mathrm{CP}]_{0}=\right.$ $200 \mu \mathrm{M}, \quad[\mathrm{DOC}]_{0}=14.4 \mathrm{ppm}, \quad\left[\mathrm{TiO}_{2}\right]=[\mathrm{ZnO}]=1.2 \mathrm{~g} \mathrm{~L}^{-1}, \quad\left[\mathrm{TiO}_{2} / \mathrm{SnO}_{2}\right]=$ $\left.\left[\mathrm{ZnO} / \mathrm{SnO}_{2}\right]=\left[\mathrm{TiO}_{2} / \mathrm{ZnO}\right]=0.6 / 0.6 \mathrm{~g} \mathrm{~L}^{-1}\right)$. 


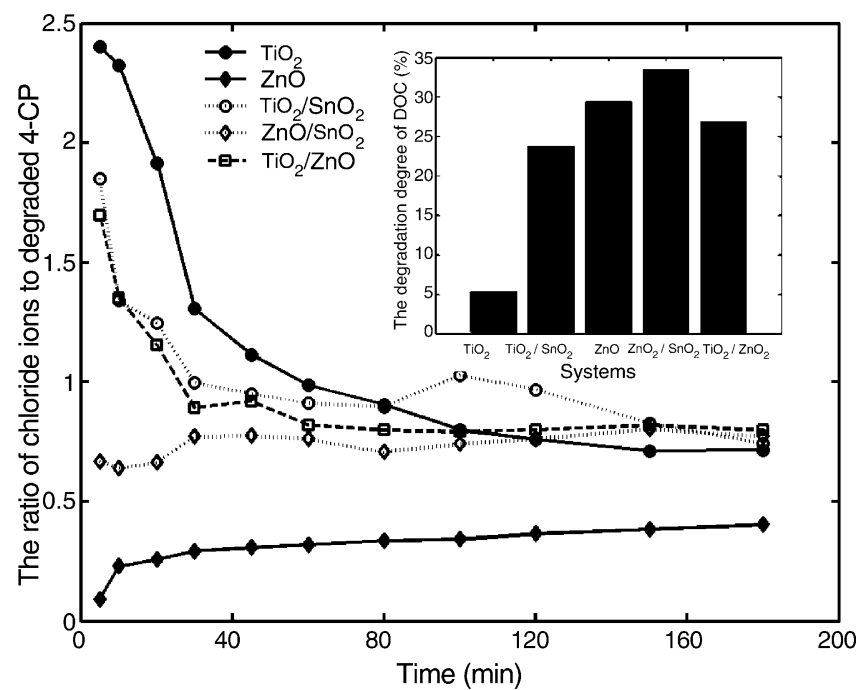

Fig. 5. The scenario of ratios of released chloride ions the degraded 4$\mathrm{CP}$ inclusive of the reduction of DOC at $\mathrm{pH} 11\left(\left[\mathrm{Cl}^{-}\right]_{\text {formed }} /[4-\mathrm{CP}]_{\text {degraded }}\right)$ $\left([4-\mathrm{CP}]_{0}=200 \mu \mathrm{M}, \quad[\mathrm{DOC}]_{0}=14.4 \mathrm{ppm}, \quad\left[\mathrm{TiO}_{2}\right]=[\mathrm{ZnO}]=1.2 \mathrm{~g} \mathrm{~L}^{-1}, \quad\left[\mathrm{TiO}_{2} /\right.\right.$ $\left.\left.\mathrm{SnO}_{2}\right]=\left[\mathrm{ZnO} / \mathrm{SnO}_{2}\right]=\left[\mathrm{TiO}_{2} / \mathrm{ZnO}\right]=0.6 / 0.6 \mathrm{~g} \mathrm{~L}^{-1}\right)$.

material. The $\mathrm{TiO}_{2}$ system reveals that the initial step was the breaking off the chlorinated function group as described above. It must rather be ascribed to the difference between the chemical and physical properties of the catalysts.

In summary, this section integrated the patterns of Figs. 3-5 to infer the simplified mechanism of 4-CP degradation in the $\mathrm{TiO}_{2}$ and $\mathrm{ZnO}$ systems. In both systems, DOC degradation occurs to a lesser extent than that of 4-CP, suggesting the presence of other organic compounds generated from 4-CP degradation. In the $\mathrm{ZnO}$ systems, the predominant intermediates are the chlorinated compounds while hydroxylation intermediates are significant in the $\mathrm{TiO}_{2}$ systems. In fact, the appearance of chloride ions does not balance the disappearance of 4-CP for each test. This fact clearly indicates that in a heterogeneous system, 4-CP degra-

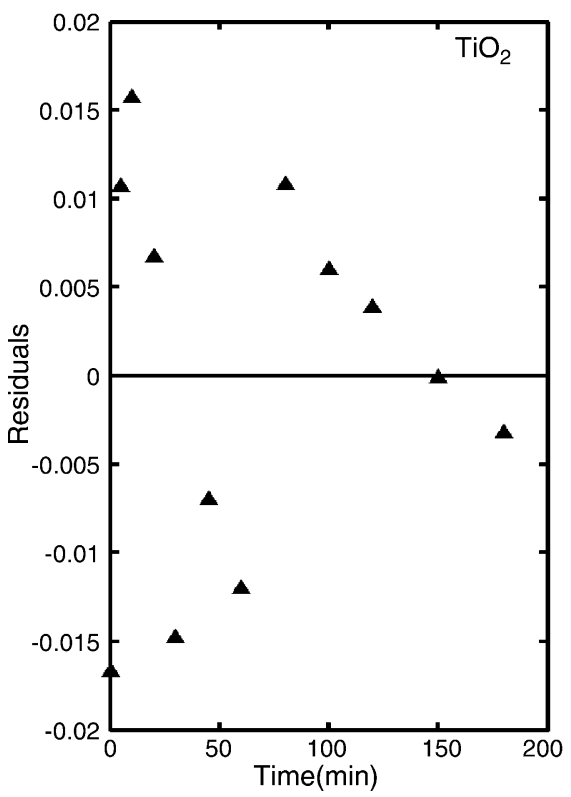

dation occurs by a mechanism that is responsible for releasing chloride ions into the solution and by a mechanism in which that release dose not occur.

\subsection{Kinetic parameters within IPET effect}

The model parameters are obtained by fitting the temporal degradation rate of 4-CP to the polynomial equation. Table 2 lists the model parameters for each set of data. Data used herein must be analyzed by estimating the observed degradation rate constants and thus introduce additional uncertainty. Therefore, some statistical parameters are used to support the validity of the model in the work. The plot of residuals for this analysis is shown in Figs. 6-8 for each system at its corresponding state, which indicate a random scatter with no discernible pattern about $\varepsilon_{i}=0$. The fit of the model to the experimental data is good $\left(r^{2}>0.9\right)$, sequentially, and all of the $t$-ratios of each parameter are exceed four, the error variances are all below some critical value. Accordingly, the proposed model is effective in determining the parameters, $\beta$ and $\gamma$.

The same electrode was used for the entire sets of experiments, so that $g_{\text {ave }}$ was theoretically a constant as the reaction proceeds in a given photocatalystic system. Therefore, $\gamma$, incorporated in the terms $g_{\text {ave }}$, depends significantly on physical and optical properties of catalysts. The $\gamma$ values in the $\mathrm{ZnO}$ system normally exceed those in the $\mathrm{TiO}_{2}$ system (Table 2), indicating that $\mathrm{ZnO}$ powders accept lower irradiation, but all of the $\mathrm{ZnO}$ systems exhibit better photocatalytic performances. The phenomenon is consistent with the aforementioned result that $\mathrm{ZnO}$ is highly photo-active to 4-CP degradation. Meanwhile, the results also present that the $\gamma$ values are explicitly higher in the coupled tests than in the single tests in the same systems (Table 2). Such a trend delivers that more catalysts can be irradiated in single tests rather than in coupled tests during the irradiation course. Perhaps, the carrier catalyst, $\mathrm{SnO}_{2}$, has the

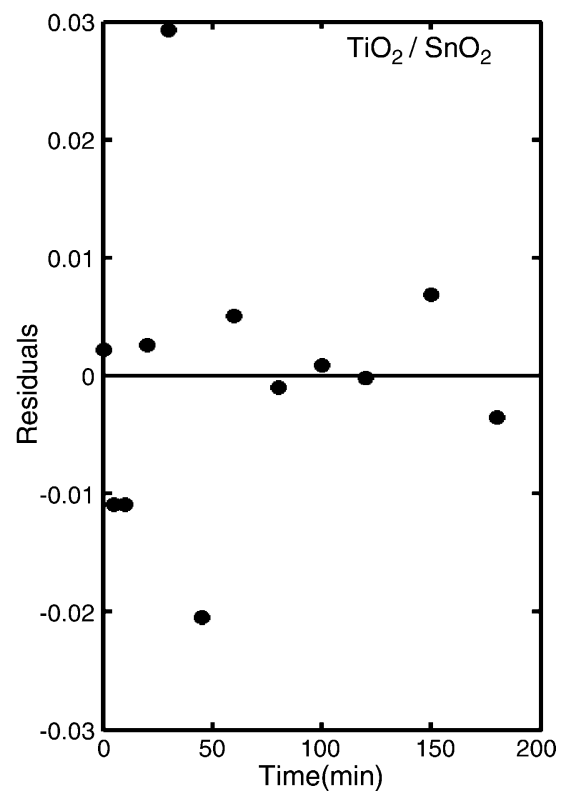

Fig. 6. The residuals resulting from the fitting analysis of the rate estimation for $\mathrm{TiO}_{2}$ systems at $\mathrm{pH} 4\left(\left[\mathrm{TiO}_{2}\right]=1.2 \mathrm{gL}^{-1},\left[\mathrm{TiO}_{2} / \mathrm{SnO}_{2}\right]=0.6 / 0.6 \mathrm{gL}^{-1}\right)$. 

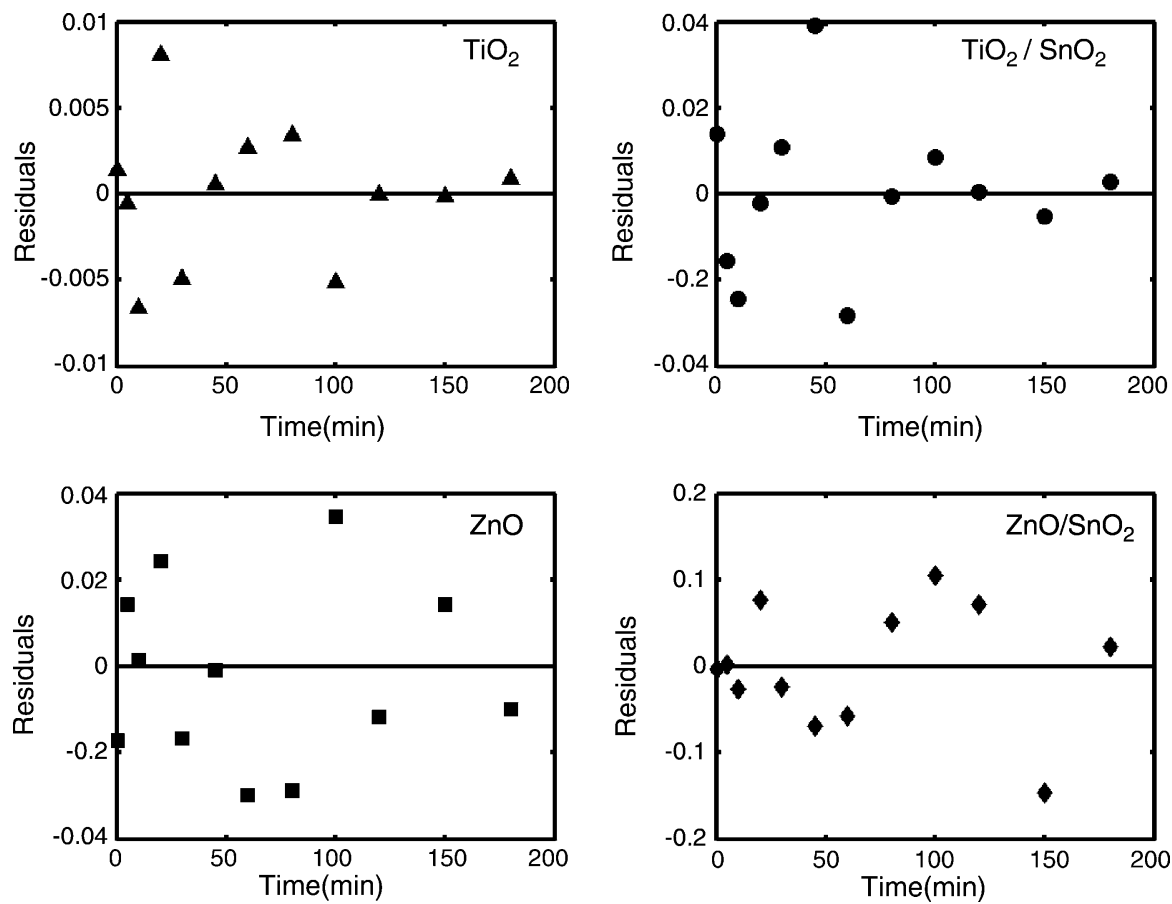

Fig. 7. The residuals resulting from the fitting analysis of the rate estimation for $\mathrm{TiO}_{2}$ and $\mathrm{ZnO}$ systems at $\mathrm{pH} 7\left(\left[\mathrm{TiO}_{2}\right]=[\mathrm{ZnO}]=1.2 \mathrm{gL}^{-1},\left[\mathrm{TiO}_{2} / \mathrm{SnO}_{2}\right]=\right.$ $\left.\left[\mathrm{ZnO} / \mathrm{SnO}_{2}\right]=\left[\mathrm{TiO}_{2} / \mathrm{ZnO}\right]=0.6 / 0.6 \mathrm{~g} \mathrm{~L}^{-1}\right)$.

shelter effects, so less irradiated catalyst is present in the coupled tests. Accordingly, such an event clarifies definitely the factor dominating the photocatalytic efficiency is the recombination opportunities rather than the amount of the irradiated photons.

Although our investigation does not address the vectorial transfer rate within the IPET process, the IPET effect could still be checked on variance of $\beta$ values for each test. Namely, $\beta$ is governed mainly by the synergy of the IPET effect and the recombination reaction. $\beta$ values are smaller in the coupled tests than in the single tests, revealing that IPET effect provides an effective approach to retard the recombination opportunities of generated photo-electrons and photo-holes. $\mathrm{ZnO}$ and $\mathrm{ZnO} / \mathrm{SnO}_{2}$ tests at $\mathrm{pH} 11$ yield $\beta$ values of $(4.660 \pm 0.346) \times 10^{-3}$ and $(3.494 \pm 0.119) \times 10^{-3}$, respectively, suggesting that the
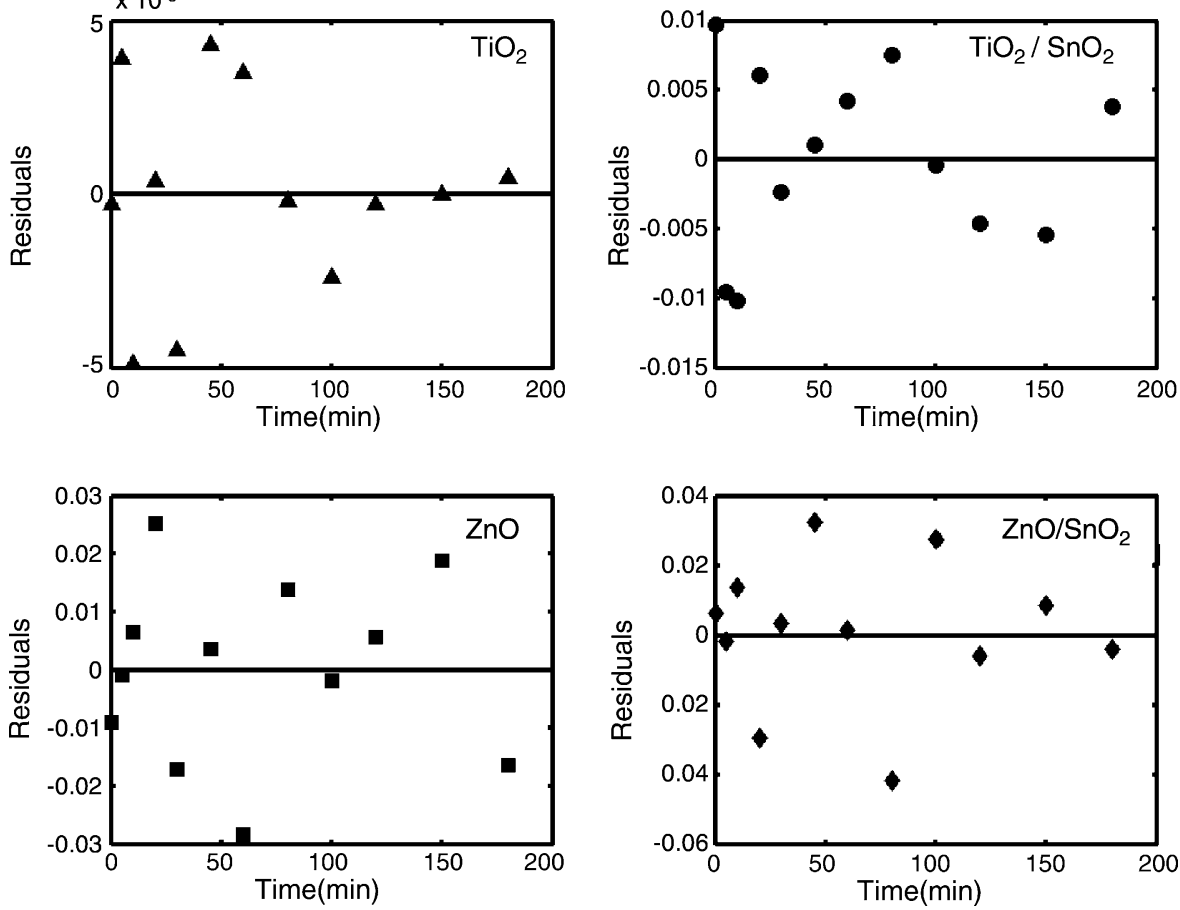

Fig. 8. The residuals resulting from the fitting analysis of the rate estimation for $\mathrm{TiO}_{2}$ and $\mathrm{ZnO}$ systems at $\mathrm{pH} 11\left(\left[\mathrm{TiO}_{2}\right]=\left[\mathrm{ZnO}=1.2 \mathrm{gL}^{-1},\left[\mathrm{TiO}_{2} / \mathrm{SnO}_{2}\right]=\right.\right.$ $\left.\left[\mathrm{ZnO} / \mathrm{SnO}_{2}\right]=\left[\mathrm{TiO}_{2} / \mathrm{ZnO}\right]=0.6 / 0.6 \mathrm{~g} \mathrm{~L}^{-1}\right)$. 
decrease in the recombination rate is around $25 \pm 3 \%$. In contrast to $\mathrm{TiO}_{2}$ and $\mathrm{TiO}_{2} / \mathrm{SnO}_{2}$ tests under the same condition, in which the recombination performance is almost $21 \pm 0.3 \%$ lower. Therefore, the $\mathrm{ZnO} / \mathrm{SnO}_{2}$ tests provide a more reliable channel to retard the recombination reaction. The same tendency can be observed at $\mathrm{pH} 7$. These results reflect that the larger energy gap causes the more efficient separation of pairs of photo-electrons and photo-holes (Fig. 1). Notably, the variance of $\beta$ values also remarkably reveals that the recombination rate is directly related to $\mathrm{pH}$ which seems to be responsible for the suppression of the recombination. However, the mechanism remains as a matter to be discussed further. Briefly, the results reveal that $\mathrm{TiO}_{2} / \mathrm{SnO}_{2}, \mathrm{ZnO} / \mathrm{SnO}_{2}$ and $\mathrm{TiO}_{2} / \mathrm{ZnO}$ can facilitate the separation of photo-electrons and photo-holes. The promotion extent of the IPET effect follows the order: $\mathrm{ZnO} / \mathrm{SnO}_{2}>\mathrm{TiO}_{2} / \mathrm{SnO}_{2}>\mathrm{TiO}_{2} / \mathrm{ZnO}$, which corresponds to the corresponding energy gap. In summary, the efficiency of the photocatalysis is strongly attributed to the suppression of the recombination of photo-electrons and photo-holes, rather than the amount of the generated photons. Additionally, the results demonstrated herein constitute reliable evidences that the IPET effect apparently provide an effective means to improve photocatalysis.

\section{Conclusions}

In the single-photocatalyst test, $\mathrm{ZnO}$ exhibited the highest catalytic activity and a better degree of reduction of DOC than other single catalyst tests at $\mathrm{pH} 7$ and 11. The observed rate constants of the coupled-catalyst systems, $\mathrm{TiO}_{2} / \mathrm{SnO}_{2}$ and $\mathrm{ZnO} / \mathrm{SnO}_{2}$, are higher than that of $\mathrm{TiO}_{2}$ and $\mathrm{ZnO}$ approximately by a factor of 1.5-3. Also, the reductions of DOC for $\mathrm{TiO}_{2} / \mathrm{SnO}_{2}$ and $\mathrm{ZnO} / \mathrm{SnO}_{2}$ tests are $8-20 \%$ higher than that of the single-photocatalyst system. One recommended course of action related to this study is to assess the recombination rate within IPET process. A comparison of the model parameters revealed significant trends that can be qualitatively related to the experimental conditions and test systems. Substantially, this study concludes that the IPET effect on the photocatalysis dose deliver the anticipated advantages based on the disappearance rate of $4-\mathrm{CP}$. The results demonstrate that the recombination rate is prone to decline in systems with a large energy gap, which dose correspond to the concept of IPET process.

\section{Acknowledgement}

The authors would like to thank the National Science Council of the Republic of China for financially supporting this research under Contract No. NSC 93-2622-E-264-004-CC3.

\section{References}

[1] M. Lewandowski, D.F. Ollis, A two-site model simulating apparent deactivation during photocatalytic oxidation of aromatics on titanium dioxide $\left(\mathrm{TiO}_{2}\right)$, Appl. Catal. B Environ. 43 (2003) 309-327.
[2] A. Sobczynski, L. Duczmal, W. Zmudzinski, Phenol destruction by photocatalysis on $\mathrm{TiO}_{2}$ : an attempt to solve the reaction mechanism, J. Mol. Catal. A Chem. 213 (2004) 225-230.

[3] K. Chiang, R. Amal, T. Tran, Photocatalytic oxidation oxidation of cyanide: kinetic and mechanistic studies, J. Mol. Catal. A Chem. 193 (2003) 285-297.

[4] P.B. Amama, K. Itoh, M. Murabayashi, Photocatalytic degradation of trichloroethylene in dry and humid atmospheres: role of gas-phase reactions, J. Mol. Catal. A Chem. 217 (2004) 109-115.

[5] C.B. Almquist, P. Biswas, Role of synthesis method and particle size of nanostructured $\mathrm{TiO}_{2}$ on its photoactivity, J. Catal. 212 (2002) 145156.

[6] J.C. Lee, M.S. Kim, B.W. Kim, Removal of paraquat dissolved in a photoreactor with $\mathrm{TiO}_{2}$ immobilized on the glass-tubes of UV lamps, Water Res. 36 (2002) 1776-1782.

[7] A.D. Paola, E. Garcia-Lopez, S. Ikeda, G. Marci, B. Ohtani, L. Palmisano, Photocatalytic degradation of organic compounds in aqueous system by transition metal doped poly crystalline $\mathrm{TiO}_{2}$, Catal. Today 78 (2002) 87-93.

[8] N. Serpone, P. Maruthamuthu, P. Pichat, E. Pelizzatti, H. Hidaka, Exploiting the interpartical electron transfer process in the photocatalysed oxidation of phenol, 2-chlorophenol and pentachlorophenol: chemical evidence for electron and hole transfer between coupled semiconductors, J. Photochem. Photobiol. A Chem. 85 (1995) 247-255.

[9] C. Wang, J. Zhao, X. Wang, B. Mai, G. Sheng, P. Peng, J. Fu, Preparation, characterization and photocata;ytic activity of nano-sized $\mathrm{ZnO} / \mathrm{SnO}_{2}$ coupled-photocatalysts, Appl. Catal. B Environ. 39 (2002) 269-279.

[10] S.C. Lo, C.F. Lin, C.H. Wu, P.H. Hsieh, Capability of coupled CdSe/TiO 2 for photocatalytic degradation of 4-chlorophenol, J. Hazard. Mater. B114 (2004) 183-190.

[11] C.H. Wu, Comparison of azo dye degradation efficiency using UV/single semiconductor and UV/coupled semiconductor systems, Chemosphere 57 (2004) 601-608.

[12] M.R. Hoffmann, S.T. Martin, W. Choi, W. Bahnemann, Environmental applications of semiconductor photocatalysis, Chem. Rev. 95 (1995) 9-96.

[13] R.B. Draper, M.A. Fox, Titanium dioxide photosensitized reactions studied by diffuse reflectance flash photolysis in aqueous suspensions of $\mathrm{TiO}_{2}$ power, Langmuir 6 (1990) 1396-1402.

[14] E.R. Carraway, A.J. Hoffman, M.R. Hoffman, Photocatalytic oxidation of organic acids on quantum-sized semiconductor colloids, Environ. Sci. Technol. 28 (1994) 1994.

[15] K.I. Ishibashi, A. Fujishima, T. Watanabe, K. Hashimoto, Quantum yields of active oxidative species formed on $\mathrm{TiO}_{2}$ photocatalyst, J. Photochem. Photobiol. A Chem. 134 (2000) 139-142.

[16] L. Sun, J.R. Bolton, Determination of quantum yield for the photochemical generation of hydroxyl radicals in $\mathrm{TiO}_{2}$ suspensions, J. Phys. Chem. 100 (1996) 4127-4134.

[17] H. Gerischer, Photocatalysis in aqueous solution with $\mathrm{small}^{\mathrm{TiO}_{2}}$ particles and the dependence of the quantum yield on particle size and light intensity, Electrochim. Acta 40 (1995) 1277-1281

[18] C.B. Almquist, P. Biswas, A mechanistic approach to modeling the effect of dissolved oxygen in photo-oxidation reactions on titanium dioxide in aqueous systems, Chem. Eng. Sci. 56 (2001) 3421-3430.

[19] Y. Meng, X. Huang, Y. Wu, X. Wang, Y. Qian, Kinetic study and modeling on photocatalytic degradation of para-chlorobenzoate at different light intensities, Environ. Pollut. 117 (2002) 307-313.

[20] R.A. Doong, C.H. Chen, R.A. Maithreela, S.M. Chang, The influence of $\mathrm{Ph}$ and Cadmium sulfide on the sulfide on the photocatalytic degradation of 2-chlorophenol in titanium dioxide suspensions, Water Res. 35 (2001) 2873-2880.

[21] Y. Ku, I.L. Jung, Photocatalytic reduction of Cr(VI) in aqueous solutions by irradiation with the presence of titanium dioxide, Water Res. 35 (2001) 135-142.

[22] A.A. Khodja, T. Sehili, J.F. Pilichowski, P. Boule, Photocatalytic degradation of 2-Phenyphenol on $\mathrm{TiO}_{2}$ and $\mathrm{ZnO}$ in aqueous suspensions, J. Photochem. Photobiol. A Chem. 141 (2001) 231-239. 
[23] C.A.K. Gouvea, F. Wypych, S.G. Moraes, N. Duran, N. Nagata, P. PeraltaZamora, Semiconductors-assisted photocatalytic degradation of reactive dyes in aqueous solution, Chemosphere 40 (2000) 433-440.

[24] B. Dindar, S. Icli, Unusual photoreactivity of zinc oxide irradiated by concentrated sunlight, J. Photochem. Photobiol. A Chem. 140 (2001) 263-268.

[25] C. Hu, Y. Tang, J.C. Yu, P.K. Wong, Photocatalytic degradation of cation blue X-GRL adsorbed on $\mathrm{TiO}_{2} / \mathrm{SiO}_{2}$ photocatalyst, Appl. Catal. B Environ. 40 (2003) 131-140.

[26] U. Stafford, K.A. Gray, P.V. Kamat, Photocatalytic degradation of 4chlorophenol: the effects of varying $\mathrm{TiO}_{2}$ concentration and light wavelength, J. Catal. 167 (1997) 25-32.

[27] H. Alekabi, N. Serpone, Kinetic studies in heterogeneous photocatalysis. 1. photocatalytic degradation of chlorinated phenols in aqueous solutions over $\mathrm{TiO}_{2}$ supported on a glass matrix, J. Phys. Chem. 92 (1988) 5726-5731.

[28] G. Alsayyed, J.C. D'Oliveira, P. Pichat, Semiconductor-sensitized photodegradation of 4-CP in water, J. Photochem. Photobiol. A Chem. 58 (1991) 99-114.
[29] T. Sehili, P. Boule, J. Lemaire, Photocatalysed transformation of chloroaromatic derivatives on zinc oxide IH: chlorophenols, J. Photochem. Photobiol. A Chem. 50 (1989) 117-127.

[30] M. Fujii, T. Kawai, S. Kawai, Photocatalytic activity and the energy levels of electrons in a semiconductor particle under irradiation, Chem. Phys. Lett. 106 (1984) 517-522.

[31] M.F. Finlayson, B.L. Wheeler, N. Kakuta, K.H. Park, A.J. Bard, A. Campion, M.A. Fox, S.E. Webber, J.M. White, Determination of flat-band position of CdS crystals, films, and powders by photocurrent and impedance techniques. Photoredox reaction mediated by intragap states, J. Phys. Chem. 89 (1985) 5676-5681.

[32] J.R. White, A.J. Bard, Electrochemical investigation of photocatalysis at CdS suspensions in the presence of Methylviologen, J. Phys. Chem. 93 (1985) 1947-1954.

[33] G. Redmond, A. O'Keefe, C. Burgess, C. MacHale, D. Fitzmaurice, Spectroscopic determination of the flatband potential of transparent nanocrystalline ZnO film, J. Phys. Chem. 97 (1993) 11081-11086. 